\title{
Propofol-induced damage to the blood-brain barrier endothelium evinces a vascular endothelial growth factor (VEGF) -dependent mechanism.
}

\author{
Dustin W. Parsons ${ }^{1}$, Jason M. Hughes ${ }^{1}$ and Scott G. Canfield ${ }^{1}$ \\ ${ }^{1}$ Indiana University School of Medicine, Department of Anatomy, Cell Biology \& Physiology
}

\begin{abstract}
Background and Hypothesis:
Propofol is an anesthetic commonly used to induce general anesthesia for a myriad of medical procedures. However, a growing corpus of evidence suggests that propofol-induced increases in VEGF may contribute to blood-brain barrier (BBB) leakiness in varying animal models. The BBB is a neurovascular structure which protects the central nervous system from pathogens, toxins, and other deleterious metabolites; therefore, considerations regarding BBB integrity in humans are indispensable to the practice of anesthesia. We hypothesize that propofol-induced $B B B$ dysfunction in human models is partially mediated by an increase in VEGF expression.
\end{abstract}

\section{Methods:}

We utilized human induced pluripotent stem cells (hiPSC) to derive brain microvascular endothelial cells (BMECs) — the barrier forming cell type of the BBB. BMECs were then subjected to clinically relevant doses of propofol for 3 hours, and barrier integrity was monitored via transendothelial electrical resistance (TEER) and para-cellular permeability for up to 72 hours. Propofol-induced VEGF levels were determined with an ELISA assay. Axitinib, a VEGF receptor blocker, was further utilized to assess the role of VEGF in propofol-induced BBB breakdown.

\section{Results:}

Prior works, including this study, have shown that propofol induces BBB damage, as demonstrated by decreases in TEER; here, preliminary work with ELISA assays further suggest that BMECs treated with propofol demonstrate an upregulation of VEGF. Pretreatment of BMECs with Axitinib before the addition of propofol partially rescues TEER and thus attenuates the propofol-mediated diminution of TEER. These observations thereby implicate VEGF as a damage mediator after propofol treatment.

\section{Conclusion and Potential Impact:}

This study utilized an in vitro model to demonstrate that propofol may mediate, in part, damage to blood- brain barrier endothelium via a VEGF dependent mechanism; thus, this work may guide future investigations to facilitate the development of safer anesthetic alternatives, or towards additional pharmacologic interventions that counteract propofol-mediated damage during anesthetic induction. 